\title{
مسيرة التقدم في أسلمة علم النفس \\ مساهمة كتاب: علم النفس التجريبي في التراث العربي الإسلامي
}

للدكتور عمر هارون خليفة*

\section{*. د. محمد مقداد}

مقدمة

إن التراث العربي الإسلامي-بما في ذلك التراث المتعلق بعلم النفس- تتنازعه اليوم ثلاثة مواقف: أولها موقف تمجيدي يرى ضرورة اعتماد التراث بما هو عليه دون نظر في مفرداته ومفاصله الأساسية باعتباره يمثل تقاليد إسلامية موروثة. وثانيهما موقف تحقيري يرفض التراث جملة وتفصيلاً باعتباره صدى لأعصر بائدة، تعكس ثقافته عصوراً لما خصوصياتما. وينزع أصحاب هذا الموقف عن المسلمين فضل السبق والإضافة في كثير من ججالات العلم. وثالث المواقف موقف يرى أفراده ضرورة احترام التراث مع تناوله بمنهج أساسه النقد والتحليل والنظرة التاريخية إلى مضمونه حتى يمكن تمييز الغث فيه من السمين. وكما هو واضح، فإن هذا الموقف يتجاوز الموقفين السابقين لأنه يأخذ بعين الاعتبار صلات التأثر والتأثير بين الثقافة العلمية العربية الإسلامية والثقافات الأخرى. وفي الواقع فإن هذا هو موقف مدرسة أسلمة المعرفة. وإذا كان لي أن أصنف كتاب الدكتور عمر خليفة في موقف من هذه المواقف الثلاثة، فلا شك سيكون موقعه في الموقف الثالث. قَدف هذه الورقة إلى عرض الكتاب أولاً والإشارة إلى مقاربات أسلمة علم النفس المختلفة بما فيها المقاربة المتبناة في هذا الكتاب.

الجمال العام للكتاب هو التراث النفسي العربي الإسلامي. ومن الممكن تقسيم هذا المجال العام إلى جالات فرعية متعددة هي المجال العلمي ويتعلق بقضايا علم النفس وموضوعاته المختلفة والمجال اللغوي ويتعلق بالتراث المكتوب باللغة العربية دون غيرها والمجال الزمني ويتعلق بالتراث الذي تم نشره في الفترة ما بين

$$
\begin{aligned}
& \text { * خليفة، عمر هارون. علم النفس التجريبي في التراث العربي الإسلامي. بيروت: المؤسسة العربية للدراسات والنشر، } 2001 \text { م. }
\end{aligned}
$$

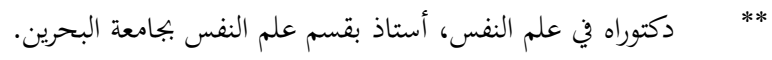


القرن التاسع الميلادي والقرن الخامس عشر الميلادي، وأخيراً بجال التراث المكتوب دون الاهتمام بالتراث المصنوع كالآلات والمكائن والمباني من مساجد وقصور وغيرها.

ويمكن تقسيم أهداف الكتاب إلى مجموعتين هما: أولاً، توسيع نطاق عمل الدكتور الزبير البشير طه1 1 في كتابه "علم النفس في التراث العربي الإسلامي" (1995) إضافة إلى إحياء مقياس ابن الهيثم للغلط البصري، ورسم ملامح علم النفس التجريبي في التراث العربي الإسلامي. وثانياً تحديد حجم التأثيرات التي أحدثها علم النفس التجربي في التراث العربي الإسلامي في تطور الحضارة العلمية والتقنية الغربية. وثمة هدف ضمني آخر يمكن استنتاجه من خلال العنوان العام للكتاب والعناوين المختلفة لفصوله هو جمع ما يمكن من التراث النفسي العربي الإسلامي وتنقيحه وعرضه للمساهمة في أسلمة علم النفس وتأصيله.

عنوان الكتاب هو: "علم النفس التجريبي في التراث العربي الإسلامي" وهو لا يختلف عن عنوان كتاب الدكتور طه الزبير إلا في إضافة كلمة التجريب. وقد بين الكاتب أن عنوانه هذا مستلهم من عنوان كتاب أستاذه، وأنه يريد مواصلة عملية البناء التي بدأها من قبله. وعن مدى ارتباط العنوان بالمتوى العام للكتاب، فقد كان يمكن أن يكون قوياً لو أن الكاتب اكتفى بالإشارة إلى علم النفس بدلاً من الإشارة إلى علم النفس برسئ التجريبي في التراث العربي الإسلامي. لأن كلمة علم نفس تجريبي توحي أن الباحث مهتم بالبحث عن فرع واحد من فروع علم النفس وهو علم النفس التجريبي دون غيره من الفروع. لكن الباحث تناول في كتابه فروعاً أخرى مثلا علم النفس العيادي الإكلينيكي في الفصل السابع وعلم النفس الفيزيولوجي في الفصل الثالث وعلم نفس الحيوان في الفصل الرابع وعلم نفس الطفل في الفصل الخامس وعلم النفس الرياضي في الفصل السابع، وعلم النفس الفيزيائي في الفصل العاشر. ويمكن اقتراح العنوان الآيت: "ميادين علم النفس في التراث العربي الإسلامي" للكتاب بدلاً من عنوانه الحالي.

\section{محتوى الكتاب}

يقع الكتاب في 444 صفحة من الحجم المتوسط، وتوزّع محتواه في اثني عشر فصلاً. ويتناول الفصل الأول (علم النفس والتراث) مجموعة من القضايا أهمها مسألة تأصيل علم النفس أو أسلمته، حيث تقاسم 
ذلك ابتحهان سماهما الباحث الاتحاه التوفيقي الذي يحاول أن يقارب بين النص المطلق (القرآن) والنص النسبي (علم النفس) والاتحاه التراثي الذي يحاول المقاربة بين التراث النفسي العربي الإسلامي وعلم النفس الغربي الحالي. وقد انتقدهما الباحث معاً وبين عدم تمكنهما الفعلي من تحقيق المقاربة المنشودة والانسيابية بين الطرفين لتغلب العمل التلفيقي على المجهودات النزيهة. وتناول الفصل الثاني علم التشريح مشيرا إلى مجهودات بعض العلماء المسلمين الأوائل من أمثال ابن سينا وابن النفيس وابن الهيثم في هذا العلم. وتناول الفصل الثالث علم النفس الفيزيولوجي عارضاً بصفة أساسية أعمال العالم ابن سينا وخاصة في كتبه المختلفة الرسالة والشفاء والنجاة والقانون. وتناول الفصل الرابع علم نفس الحيوان مقدماً تاريخ هذا العلم عند علماء النفس كإس العرب ومصادره وموضوعاته ومناهج البحث فيه. كما تناول مقارنة بين دراسات الجاحظ وسكنر للحيوانات والطيور. وتناول الفصل الخامس علم نفس الطفل مبرزاً مجهودات بعض العلماء المسلمين من أمثال ابن الجزار القيروائ والبلدي في هذا المجال. وتناول الفصل السادس علم النفس السينوي مبينا تنوع مداخله (المدخل الميتافيزيقي والذهني المعريز والتجريبي) ومناهج البحث فيه وتأثيره في علم النفس الحديث. وتناول الفصل السابع علم النفس المرضى مبرزاً فيه مجهودات الباحثين المسلمين الأوائل من أمثال ابن سينا والرازي وغيرهما سواء في تشخيص الأمراض النفسية وتصنيفها أو في علاجها وظيفيا وباستخدام الأدوية. وتناول الفصل الثامن البيمارستانات العربية أو العيادات النفسية في الحواضر العربية في القرون الأولى، متعرضاً إلى ما كان يتم فيها من ممارسات علاجية للمرضى النفسيين من جهة، وتدريب للمعالجين النفسيين من جهة أخرى. وتناول الفصل التاسع الرياضيات وعلم النفس عارضاً فيه بجهودات العلماء المسلمين الأوائل من أمثال الخوارزمي ونصير الدين الطوسي وابن الهيثم وابن سينا وغيرهم في الحساب والجبر والهندسة وعلم الفلك وعلم البصريات. وتناول الفصل العاشر ابن الهيثم وثورة السيكوفيزيقا وهو يختلف عن باقي الفصول الأخرى، في أن هذا الفصل خصص كاملاً للعمل الذي قام به ابن الهيثم في مجال البصريات. وتناول الفصل الحادي عشر لرّ تجارب ابن الهيثم عن الغلط البصري وهو مثله مثل الفصل السابق خاص بعرض التجارب التي قام بها ابن الهيثم في مجال أخطاء الإدراك أو الغلط البصري. وتناول الفصل الأخير أو الثاني عشر تاريخ علم النفس التجريبي في التراث العربي الإسلامي مبرزاً فروع هذا العلم وملامحه المختلفة وما يمكن أن يستفاد منه من 
تقسم الكتب العلمية عادة إلى قسمين هما: الكتب المدرسية Textbooks وكتب البحث Research books بصورة واضحة جداً كتاب بحثي كان نتيجة الجهود التي بذها الباحث في بحث التراث العربي الإسلامي وغربلته لسنوات طويلة وهذا النوع من الكتب يحتاج إلى مجهود أكبر مما قد تتناج إليه الكتب الأخرى لأن البحث عن التراث والتأكد من صحته وبحثه عمليات شاقة جداً ومكلفة ولا بد لها من الصبر والمثابرة.

وفيما يخص تسلسل فصول الكتاب، لا بد من الإشارة أولاً إلى أن فصوله المختلفة لم تكن متسلسلة التسلسل المنطقي المطلوب كما أن الباحث لم يوضح في الكتاب الأساس الذي تم الاعتماد عليه في تصنيف تلك الفصول. فمثلاً، الفصل الثالث يتعلق بمجهودات ابن سينا في ميدان علم النفس الفيزيولوجي كما أن الفصل السادس يخص كله علم النفس السينوي، وكان يمكن أن يسبق الفصل الثالث لأنه أكثر عمومية منه. وثانياً، أن الفصول يمكن تقسيمها إلى ثلاث مجموعات بحيث تكون فصول كل مجموعة مع بعضها البعض. هذه المجموعات هي: مجموعة الفصول التي تمثل فروع علم النفس المختلفة وبجموعة الفصلين الخاصين بأعمال أبن الهيثم وأخيراً مجموعة الفصول العامة وهي الفصل الأول (علم النفس والتراث) والفصل الثاني عشر (تاريخ علم النفس التجريبي في التراث العربي الإسلامي).

وقد أثبت الباحث لكل فصل مراجعه الخاصة به وهذه المراجع كثيرة جداً ومتنوعة بين المصادر الأولية والثانوية وبين المراجع العربية والأجنبية. وبين القديمة والحديثة. وهنا لا بد من التنويه باستغلال الباحث الجيد لمذه المراجع كلها والاستفادة منها. لا بد من الإشارة إلى أن الكتاب تضمن أشكالاً تخطيطية مأخوذة من كتب التراث. وعلى سبيل المثال، فقد تضمن الفصل الثاني (علم التشريح) رسماً يوضح تشريح العين وهو مأخوذ من كتاب ابن الهيثم، وررماً آخر لتوضيح تشريح العين وهو مأخوذ من كتاب كمال الدين الفارسي ورسماً ثالثاً يوضح البنية العضوية عند منصور بن الفقيه. كما عرض الفصل صوراً لأدوات التشريح التي كانت تستخدم آنذاك. 
تتمثل أزمة علم النفس في العالم العربي والإسلامي في أنه يعيش منذ سنوات عديدة بدون روح، لأن ما

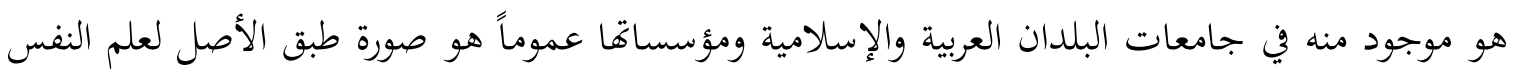
الموجود في العالم الغربي (جامعات ومؤسسات) (أنظر توفيق 1998 وحجازي 2001). استمر علم النفس يدرس فقط الجوانب التي استبقاها علماء النفس وعلى رأسها الجانب البيولوجي الفيزيقى. أما الجوانب الأخرى كالجانب المعريف والجانب الوجداني والجانب الروحي، فهي ألغيت أو تم قميشها واعتبرت قليلة الشان والأهمية. لكن في العقدين الأخيرين من القرن العشرين، فإن علم النفس قد بدأ يستعيد روحه لأن علماء النفس الجدد كالمعرفيين والإنسانيين كانوا أقل شططاً في نظرقم للإنسان واستعادوا للإنسان بعض مفقوداته. لكن كيف عادت الجوانب المفقودة؟ للإجابة عن هذا السؤال، تلزم الإشارة إلى تطور مدارس علم النفس المختلفة وتطور نظرتا للإنسان.

أولاً: كانت مدرسة التحليل النفسي Psychoanalysis مثثلة في فرويد (1856م - 1939م)

والتي نشأت سنة 1895م والسلوكية مثلة في سكنر (1904م-1990م) والتي نشأت سنة 1913م متطرفتين جداً في نظرتما للإنسان. في هاتين المدرستين كانت الجوانب البيولوجية سواء المنظورة (المدرسة السلوكية) أو غير المنظورة (المدرسة التحليلية) هي الجوانب التي تم أخذها بعين الاعتبار فقط. ولم تعر المدرستان أية أهمية تذكر لحرية الفرد. فالإنسان في رأيهما مجبر وليس مختاراً. ففرويد مثلاً يعتقد أن الفرد وكل ما يفعله مقيد ومحدد بغريزتي الحياة والموت (Freud, 1936). أما بالنسبة لسكنر، فان الفرد مثله مثل الآلة أو الماكينة المبرجة والتي لا يصدر عنها أي شئ بمحض إرادمّا. فهو أي الإنسان مستجيب طوال حياته للمثيرات التي يعج بها المحيط الذي يتواجد فيه (Skinner, 1953).

ثانياً: كانت المدرستان المعرفية مثثلة في كيللي (1905م- 1967م) والتي نشأت في الخمسينيات من القرن العشرين، والإنسانية مثثلة في ماسلو (1908م - 1970م) والتي قد نشأت في نفس الفترة أيضاً أكثر إنسانية من المدرستين سالفتي الذكر، ذلك أن كليهما (المدرستان المعرفية والإنسانية) تأخذ بعين الاعتبار دور الجوانب المعرفية والشعورية في تحريك سلوك الفرد وتوجيهه. علاوة على ذلك، فإن كليهما يؤكد على الطبيعة الخيرة للإنسان وعلى حريته. فعلى سبيل المثال، فإن كيللي متفائل حول الطبيعة الإنسانية ومؤمن بنموها 
وتطورها المستمر. كما يرى أن الإنسان يختار فيما يفعل وليس مجبرا (Kelly,1969). كما أن ماسلو مثل كيللي- متفائل حول الطبيعة الإنسانية مؤمن بنموها وتطورها المستمر. كما يرى أن الإنسان مختار فيما يفعل وليس مجبراً (Maslow, 1986).

ثالثاً: أن مدرستي علم النفس لما وراء الشخصي (Transpersonal Psychology) مثثلة في جمعية علم النفس ما وراء الشخصي (A.T.P) وعلم النفس الصوفي (Sufi Psychology) ممثلة في جمعية علم النفس الصوفي (Sufi Psychology Association) وقد نشأتا في الثمانينيات من القرن العشرين، لم تأخذا بعين الاعتبار جوانب الإنسان البيولوجية والمعرفية والوجدانية فقط، ولكن أيضاً الجوانب الروحية. فعلم النفس ما وراء الشخصي يهتم بتكامل الخبرات النفسية (المعرفية والوجدانية) والروحية. أما علم النفس الصوفي فهو يحاول احتواء علم النفس الغربي والسمو عليه بعدم تركيزه فقط على الجوانب المادية والبيولوجية والمعرفية للفرد، لكن بإعطاء أهمية كبرى ومركزية للوجدان والروح. والمدرستان سالفتا الذكر تعتبران أن الإنسان يتواجد في موقع بين الملائكة والحيوان مع إمكانية الاقتراب من هذا الطرف أو ذاك. وهما تحاولان مساعدة الإنسان في التغلب على نقائصه لتسنّم مرتبة الملائكة.

وعلى الرغم من أن التطور الذي شهده علم النفس في الموضوع والأهداف يمشي في نفس الطريق الذي تمشي فيه حركة أسلمة المعرفة التي يرفع شعاراتحا كثير من المؤسسات العلمية الإسلامية وعلى رأسها المعهد العالمي للفكر الإسلامي International Institute of Islamic Thought) في أمريكا، ${ }^{2}$ إلا أن المستوى الذي تم الوصول إليه ليس هو المستوى المنشود. لأن الروحية (Spirituality) التي طرحتها المدرستان سالفتا الذكر (مدرسة علم النفس ما وراء الشخصي ومدرسة علم النفس الصوفي) لا تبدو منسجمة مع الروحية في الدين الإسلامي. وإن المطلع على تراث بعض هاتين المدرستين (انظر مثلاً، Shafranske and Malony, 1990; Chandler et at, 1992; Lukens, 1992; (and Hinterkopf, 1994

من هذه المؤسسات أيضاً يمكن الإشارة إلى المعهد العلمي للفكر والحضارة الإسلاميتين ( Institute of Islamic Thought

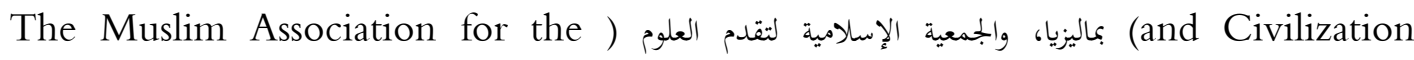
بأمريكا. (Advancement of Science 
التي تنشدها وتسعى إلى بلوغها الديانات الأرضية كالبوذية والهندوكية وغيرها من جماعات غلاة الصوفية والجماعات الطقوسية (Cults). والتي عادة ما يقصد بها التجربة الفردية التي يشعر فيها الفرد أن ذاتيته قد بتحاوزت الحدود العادية وتوحدت مع الكون أو كما يسميها غلاة الصوفية الحلول؛ حيث يؤمن بعض المتصوفة أن الله سبحانه وتعالى يحل في أجساد بعض الخاصة من عباده من الأولياء (سبحانه وتعالى عما يقولون علواً كبيرا) (الإسراء: 43).' وأحياناً أخرى يسموها الاتحاد وهو شكل من أشكال اتصال الله سبحانه وتعالى بالمتصوفة الذين يؤمنون أن ذات الله تتحد بذات الإنسان دون الحلول فيها. ومن هنا يمكن القول إن علم النفس في صورته الحالية لايزال في حاجة إلى كثير من الجهد حتى يصل إلى ما يجب أن يصل

جهود الأسلمة المبذولة من طرف علماء النفس العرب والمسلمين: يمكن تمييز ثلاث مقاربات في

سلسلة الجهود المبذولة لأسلمة علم النفس وهي:

مقاربة الإقحام أو التزيين: علماء النفس المنتمون لهذه المقاربة غالباً ما نلاحظ في منشوراتم محتوى (موضوعات ومناهج) يشبه إلى حد كبير المتوى الذي نجده في الكتب التي ينشرها الغربيون. مع فارق واحد يتمثل في أن منشورات علماء النفس العرب والمسلمين عادة ما تكون مزينة بآيات قرآنية وأحاديث نبوية شريفة وبعض أعمال العلماء المسلمين الأوائل من أمثال ابن سينا والفارابي والغزالي وغيرهم. كأمثلة لهذه المقاربة يمكن الإشارة إلى: عزت الطويل 1977 ونبيل السمالوطي 1986 وعثمان نجاتي 1982.

مقاربة بحث التراث: علماء النفس المنتمون لمذه المقاربة يحاولون تسليط الضوء على مساهمة العلماء المسلمين الأوائل التي أهملت وكادت أن تضيع، وذلك على الرغم من أصالتها وقيمتها العلمية. علاوة على هذا، فإن هؤلاء العلماء يعتبرون هذه المقاربة خطوة ضرورية لتحقيق الأسلمة في العصر الحاضر، لأن ما قام به السلف الصالح يعتبر أسلمة خالصة للعلوم الشرقية والغربية التي تواجدت في القرون الرابع والخامس والسادس الهجرية. ومن الضروري دراسة تلك التجربة الرائعة واستخلاص الدروس والعبر منها لأن حياة هذه

3

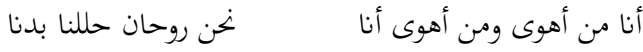

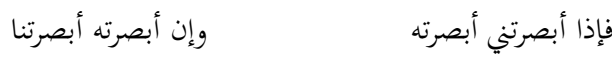


الأمة سلسلة متصلة الحلقات، لا يبنى مستقبلها إلا في ضوء الحاضر ومقومات الماضي. كأمثلة لهذه المقاربة يمكن الإشارة إلى الربيع 1993 والنجاتي والسيد 1996 وطه 1995 والعاني 1998 والخليفة 2001.

مقاربة الإتقان: علماء النفس المنتمون لهذه المقاربة يعملون تقريباً على أسلمة علم النفس وفقا للمنهجية التي قدمها المعهد العالمي للفكر الإسلامي والتي تختصر في الخطوات الثلاث: أولاً: إتقان الاختصاص. وثانياً: إتقان العلوم الإسلامية. وثالثاً: إنتاج العلم المؤسلم (Safi 1993). لهذا فهم يحاولون إتقان علم النفس وخاصة بجال الاختصاص بصورة جيدة. وبتعبير آخر، فإن هؤلاء الباحثين يتدربون بما فيه الكفاية على علم النفس وخاصة أصوله الفلسفية ومناهج البحث فيه وموضوعاته المختلفة. كما يحاولون وفى الوقت نفسه إتقان العلوم الإسلامية بالتدريب على علوم القرآن والحديث والفقه وأصول الفقه والتراث الإسلامي. بعد إتقان الجانبين سالفي الذكر والتمكن منهما، يجد الباحثون أنفسهم في موقف يسمح لهم برؤية ما في علم النفس من نقائص والرغبة في العمل على إكمالها. وبالتالي إنتاج علم النفس الإسلامي. كأمثلة لمذه المقاربة يمكن الإشارة إلى 1996 (Badri (1996), Badri (1997), and Zafar).

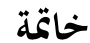

في الأخير، يجد المرء نفسه مضطراً إلى القول إن المقاربات الثلاث سالفة الذكر حلول مؤقتة للمشاكل التي يطرحها تحويل العلوم الغربية واستيرادها إلى العالم العربي والإسلامي. والحقيقة أن الأسلمة الفعلية والنهائية لا تتحقق إلا عندما تتمكن الأمة الإسلامية من بناء حضارة قوية بتعلها تنتج المعرفة التي لا تحتاج إلى أية أسلمة، وتستغني بالتالي عن استيراد المعرفة التي تجبرها على ممارسة كل الحلول المؤقتة التي تجرب حاليا. وفيما يخص مقاربة بحث التراث، إنه وعلى الرغم من أن التجذر في التراث العربي الإسلامي، شرط أساسي في أي مشروع تنموي، وخاصة في مشروع أسلمة المعرفة، إلا أنه بذاته غير كافٍ. فمشروع أسلمة العلوم لا يمكن تحقيقه بمجرد العودة إلى التراث والتنقيب فيه. 


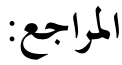

• بدري، مالك. حكمة الإسلام في تحريم الخمر. هرندن، أمريكا: المعهد العالمي للفكر الإسلامي، .1996

• خليفة، عمر هارون. علم النفس التجريبي في التراث العربي الإسلامي. بيروت: المؤسسة العربية

$$
\text { للدراسات والنشر، } 2001 .
$$

• الربيع، محمد. التراث النفسي للعلماء المسلمين الأوائل. الإسكندرية: دار المعارف الجامعية،

• السمالوطي، نبيل. الإسلام وقضايا علم النفس المعاصر. الجزائر: ديوان المطبوعات الجامعية.

• طه، الزبير البشير علم النفس في التراث الإسلامي. السودان: جامعة الخرطوم، 1995.

• الطويل، عزت. دراسات نفسية وتأملات قرآنية. الإسكندرية: دار نشر الثقافة، 1977.

$$
\text { • نجاتي، عثمان. القرآن وعلم النفس. القاهرة: دار الشروق، } 1982 .
$$

• نجاتي، عثمان والسيد محمد. علم النفس في التراث الإسلامي. القاهرة: المعهد العالمي للفكر

$$
\text { الإسلامي، } 1996 .
$$

• العاني، نزار. الشخصية الإنسانية في التراث الإسلامي. الأردن: المعهد العالمي للفكر الإسلامي،

-Badri, M. The AIDS Crisis: An Islamic Socio-cultural Perspective. Kuala Lumpur, Malaysia: IIITC, (1997). 
- Chandler, C. Holden, J.M., and Kolander, C. Counseling for Spiritual Wellness: Theory and Practice. Journal of Counseling and Development, (1992), 71, 168-175.

- Freud, S. The Ego and the Mechanisms of Defense. London: Hogarth Press, (1936).

-Hinterkopf, E. Integrating Spiritual Experiences in Counseling. Counseling and Values, (1994), 38, 165-175.

-Kelly, G. Clinical Psychology and Personality. New York: Wiley, (1969).

-Khaleefa, O. The Imperialism Of Euro-American Psychology In A Non-Western Culture: An Attempt Towards An Ummatic Psychology. The American Journal of Islamic Social Sciences, (1997), 14, 44-69 .

- Lukens, L. Focusing: Another Way To Spirituality. The Folio: A Journal for Focusing and Experiential Therapy, (1992) 10, $65-72$.

- Maslow, A. Towards a Psychology of Being. New York: Van Nostrand, (1968).

-Safi, L. The Quest For An Islamic Methodology: The Islamization Of Knowledge Project In Its Second Decade. 
The American Journal of Islamic Social Sciences, (1993) 10, 23-48 .

-Shafranske, E.P. and Malony, H.N. Clinical Psychologists' Religious And Spiritual Orientations And Their Practice Of Psychotherapy. Psychotherapy, (1990), 27, 231-241.

-Skinner, B.F. Science and Human Behavior. New York: Free Press, (1953).

-Zafar, I. Teachers Training: The Islamic Perspective. Islamabad, Pakistan: IIIT, (1996). 